\title{
Current-Induced Chiral Phase Transition in a Josephson-Junction Array
}

\author{
M.V. Simkin \\ Department of Physics, Brown University, \\ Providence, RI 02912-1843
}

\begin{abstract}
It is predicted that the fully frustrated Josephson-junction array on a square lattice with alternating columns of junctions having a reduced critical current $I_{\text {weak }}=\mu I_{0}$ (with $\mu<1$ and $I_{0}$ the critical current of all other junctions) in certain range of $\mu$ will under increasing of the external current first change from the state with zero chirality to the state with nonzero one at the lower critical current $I_{c 1}$ before entering nonsuperconducting state at the upper critical current $I_{c 2}$.

PACS numbers: 74.50.+r, 74.60.Ge, 74.60. Jg
\end{abstract}

A square two-dimensional periodic Josephson-junction array (JJA) in a uniform transverse magnetic field, with half a flux quantum per plaquette, is a realization of a fully frustrated (FF) XY model [1]. Its ground state is a checkerboard pattern of plaquettes with currents flowing clockwise and anti-clockwise [2]. The chirality, defined as the sum of currents around plaquette in a clockwise direction, has antiferromagnetic order. An interesting generalization of the FF $\mathrm{XY}$ model was proposed by Berge et al[3]. For the case of JJA it corresponds to an array in which the critical currents of alternate columns are reduced $I_{\text {weak }}=\mu I_{0}$, where $I_{0}$ is the critical current of all other junctions, and $\mu<1$. See Fig.1. For $\mu<1 / 3$ the ground state has zero chirality, while for $\mu>1 / 3$ it has a nonzero chirality. In this article I investigate the transformation of the ground state under an external current applied in the direction transverse to the weak bonds.

When the zero current ground state for $\mu<1 / 3$ has zero chirality one expects that when we apply the external current the chirality will remain zero. The ground state with current will look like Fig.1 with all $\theta=0$, except there will be a phase difference $\phi$ at each horizontal junction to accommodate current $I=I_{0} \sin \phi$. However, for big enough current this state may become unstable against fluctuations leading to nonzero chirality. To check this we write down the energy per plaquette as a function of deviations $\theta_{i}$ from the ground state 
values of phases of the superconducting nodes [4]:

$$
E=-\cos \left(\phi+\theta_{1}-\theta_{2}\right)-\cos \left(\phi+\theta_{3}-\theta_{4}\right)-\cos \left(\theta_{1}-\theta_{3}\right)+\mu \cos \left(\theta_{2}-\theta_{4}\right) .
$$

After straightforward but tedious algebra [5] we find that the nonchiral state is stable for currents $I<I_{c 1}$, given by

$$
I_{c 1}=\frac{1}{1-\mu} \sqrt{1-2 \mu-3 \mu^{2}}
$$

As we expect, $I_{c 1}(\mu=0)=1$, as we than effectively have an unfrustrated array, and $I_{c 1}(\mu=1 / 3)=0$, as for $\mu>1 / 3$ even the zero current ground state is chiral.

We now determine the upper critical current, i.e. the maximum current the array can sustain with zero voltage, but allowing for nonzero chirality, following the method of Benz et al [6] for a uniform $(\mu=1)$ array.

A chiral current-carrying state is shown in Fig.2. The phase differences across the junctions $\alpha, \beta, \gamma, \delta$ obey the following conditions. The sum of currents flowing into a node is zero:

$$
\sin \alpha-\sin \gamma-2 \sin \beta=0 .
$$

Net current in vertical direction is zero:

$$
\sin \beta=-\mu \sin \delta
$$

The sum of phase differences around plaquette is zero:

$$
\alpha-\gamma+\beta+\delta=0
$$

The net current per junction in the horizontal direction is

$$
I=\frac{1}{2}(\sin \alpha+\sin \gamma)
$$

From (3)-(6) we obtain:

$$
I^{2}=\frac{a^{2}-2 a x}{a^{2}-2 a x+x} x,
$$

where $a=\frac{1}{2}\left(\frac{1}{\mu}+1\right), x=\cos ^{2}\left(\frac{\alpha-\gamma}{2}\right)$. We now maximize $I$, given by (7), with the restriction $x \leq 1$. Straightforward, though tedious, analysis shows that for $\mu>\mu_{c}=0.2137 \ldots$ [7] the maximum current $I_{c 2}$ is achieved for some value of $x<1$ and is given by

$$
I_{c 2}=\frac{1}{2} \frac{\frac{1}{\mu}+1}{\sqrt{\frac{1}{\mu}+1}+1} .
$$

For $\mu=1$ Eq. (8) gives the result of Benz et al[ 6$] I_{c 2}=\sqrt{2}-1$. For $\mu<$ $\mu_{c}$, the maximum $I$ is achieved for $x=1$, or $\alpha=\gamma$ which is a nonchiral state. Correspondingly for $\mu<\mu_{c}, I_{c 2}=I_{c 1}$. 
In Fig.3 the phase diagram of the nonuniform FF JJA in the $(\mu, I)$ plane is shown. We see that for $0.2137 \ldots=\mu_{c}<\mu<1 / 3$ the array as the current is increased will first change from a nonchiral to a chiral state at $I=I_{c 1}$, and then to a non-superconducting state at $I=I_{c 2}>I_{c 1}$.

From Eqs. (2) and (7) we obtain for current $I$ just above $I_{c 1}$ the chirality

$$
\chi=\sin \alpha-\sin \gamma+\sin \beta-\mu \sin \delta,
$$

behaves as

$$
\chi \sim\left(I-I_{c 1}\right)^{\frac{1}{2}}
$$

I observed the above transition in computer simulations (see Fig.4). For the simulations I used, following Falo et al [8], a resistively shunted Josephsonjunction array, with each superconducting node having a capacitance to the ground. The current was injected into $16 \times 16$ array from superconducting bars at the edges. Free boundary conditions where used in the transverse direction. Very small thermal fluctuations $\left(10^{-8}\right.$ in units of Josephson energy $\left.\hbar I_{0} / 2 e\right)$ are introduced to drive the system away from the unstable nonchiral state and to check the chiral state for stability. In Fig. 4(a),(b) the phases of the superconducting nodes for the different applied currents are represented by arrows. In Fig. 4 (a) $\mu=0.32<1 / 3, I=0.3<I_{c 1}$, and the phases in one column are identical which is expected for zero chirality. In Fig. 4(b) the current $I=0.5>I_{c 1}$, the phases in a column are varying which indicates a finite chirality, which is shown in Fig. 4(c).

In conclusion I have obtained the phase diagram of nonuniform fullyfrustrated Josephson array in the applied current $(I)$-weak bond $\operatorname{strength}(\mu)$ plane. For some range of $\mu$ it implies a transition from nonchiral to chiral state under increasing of the applied current. I have observed this transition in numerical simulations.

I am grateful to N.Akino, J.M. Kosterlitz, and J.B. Marston for useful conversations. This work was supported by National Science Foundation Grant No. DMR-9222812.

\section{References}

[1] J.Villain, J.Phys.C 10,1717(1977).

[2] S.Teitel and C.Jayaprakash, Phys. Rev. Lett. 51, 1999(1983).

[3] B.Berge,H.T. Diep, A. Ghazali,and P. Lallemand, Phys. Rev. B 34, 3177 (1986).

[4] In the following I take into account magnetic field (frustration) by changing the sign of the critical current of the weak junctions. 
[5] I just expand $E$ in $\theta$ to the second order and check whether the resulting quadratic form is positive definite.

[6] S.P.Benz, M.S. Rzchowski, M. Tinkham, and C.J. Lobb, Phys. Rev. B 42 6165 (1990).

[7] exact value is $\mu_{c}=3\left(2(19+3 \sqrt{33})^{\frac{1}{3}}+2(19-3 \sqrt{33})^{\frac{1}{3}}+5\right)^{-1}$.

[8] F.Falo, A.R. Bishop, and P.S. Lomdahl, Phys. Rev. B 41, 10893 (1990). Addition of capacitances to the ground introduced here substantially simplifies the algorithm in comparision to the resistively-shunted junction model. 
Figure 1: Nonuniform fully-frustrated Josephson-junction array in a nonchiral current-carrying state. Solid lines are junctions with strong critical current, $I_{0}$. Dashed lines - those with weak critical current $\mu I_{0}$. Arrows - directions of currents. The phases of the superconducting nodes are indicated. In a nonchiral state all $\theta=0$, though this state may be unstable against fluctuations of $\theta$.

Figure 2: Nonuniform fully-frustrated Josephson-junction array in a chiral state. Thick lines with arrows - directions of currents. $\alpha, \beta, \gamma$, and $\delta$ - phase differences across junctions.

Figure 3: Phase diagram of the nonuniform fully-frustrated Josephson-junction array in the $(\mu, I)$ plane, computed using Eqs.(2) and (8).

Figure 4: Results of numerical simulations for a $16 \times 16$ array with the weak bonds strength $\mu=0.32$. In (a) and (b) phases of superconducting nodes are represented by arrows. (a) corresponds to current $I=0.3$ and is in a nonchiral part of the diagram (see Fig.3). (b) $I=0.5$, which is bigger than the critical current for the chiral transition. (c) is (b) in chirality (sum of currents around the plaquette) representation. 


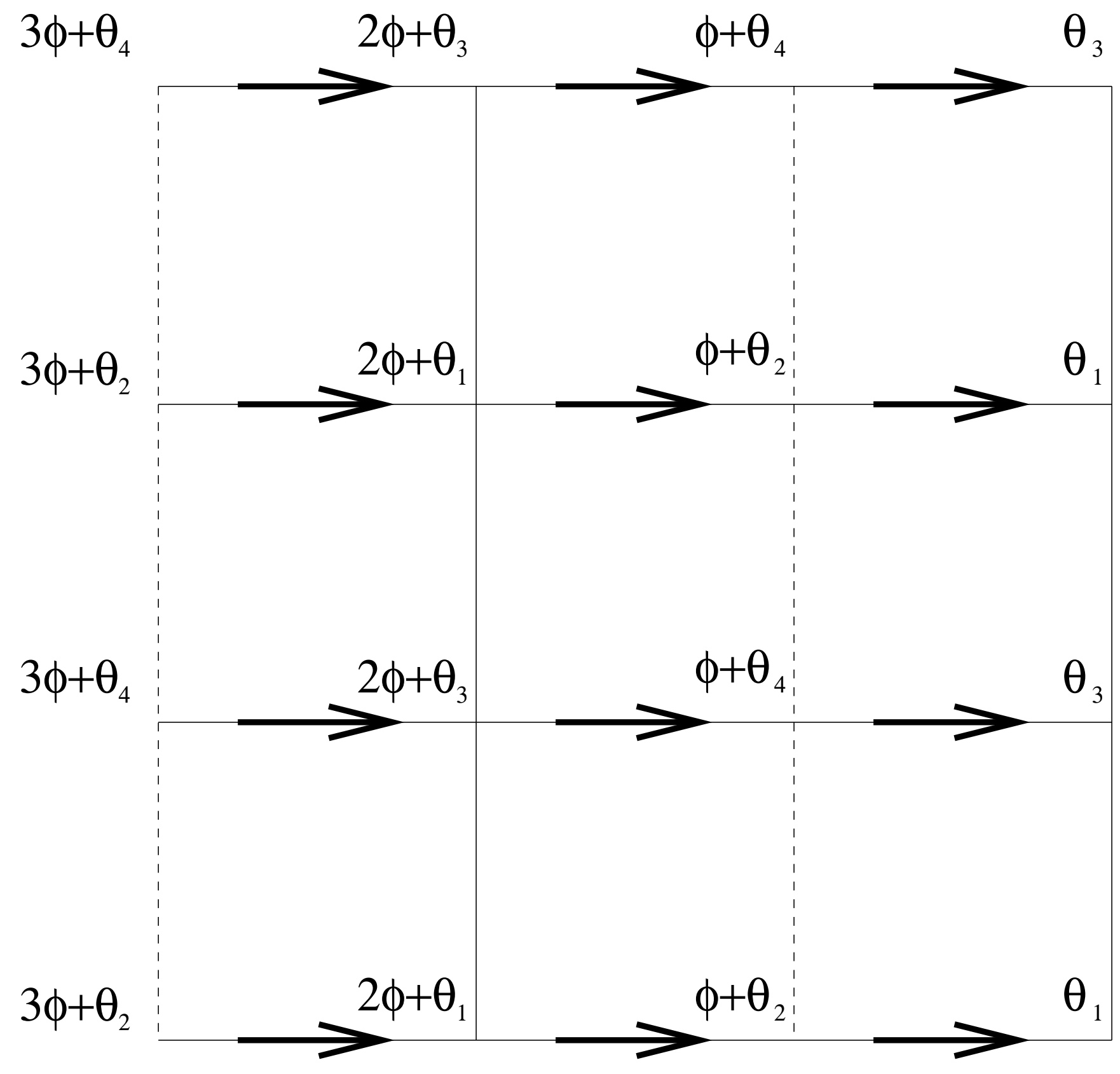




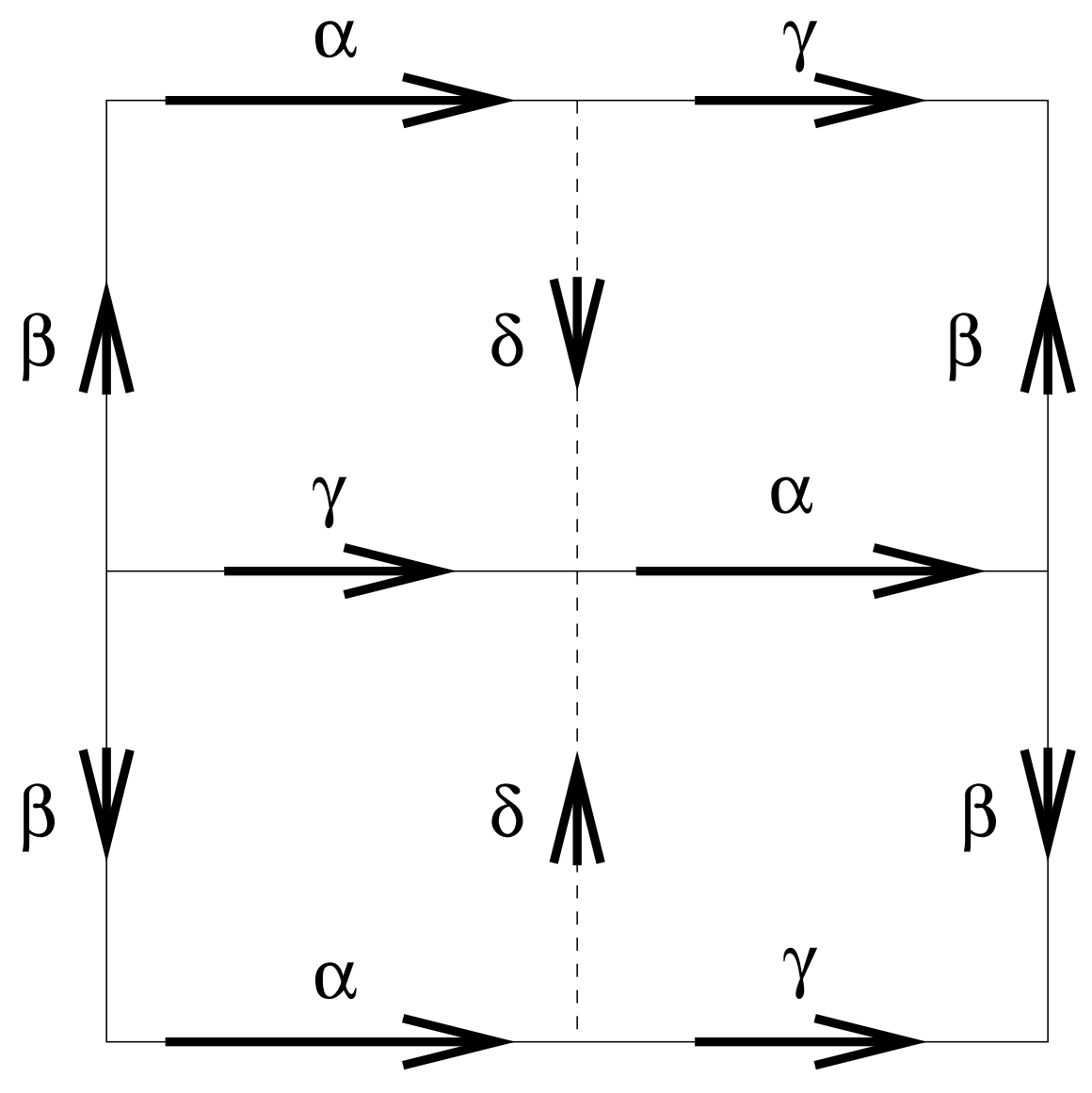




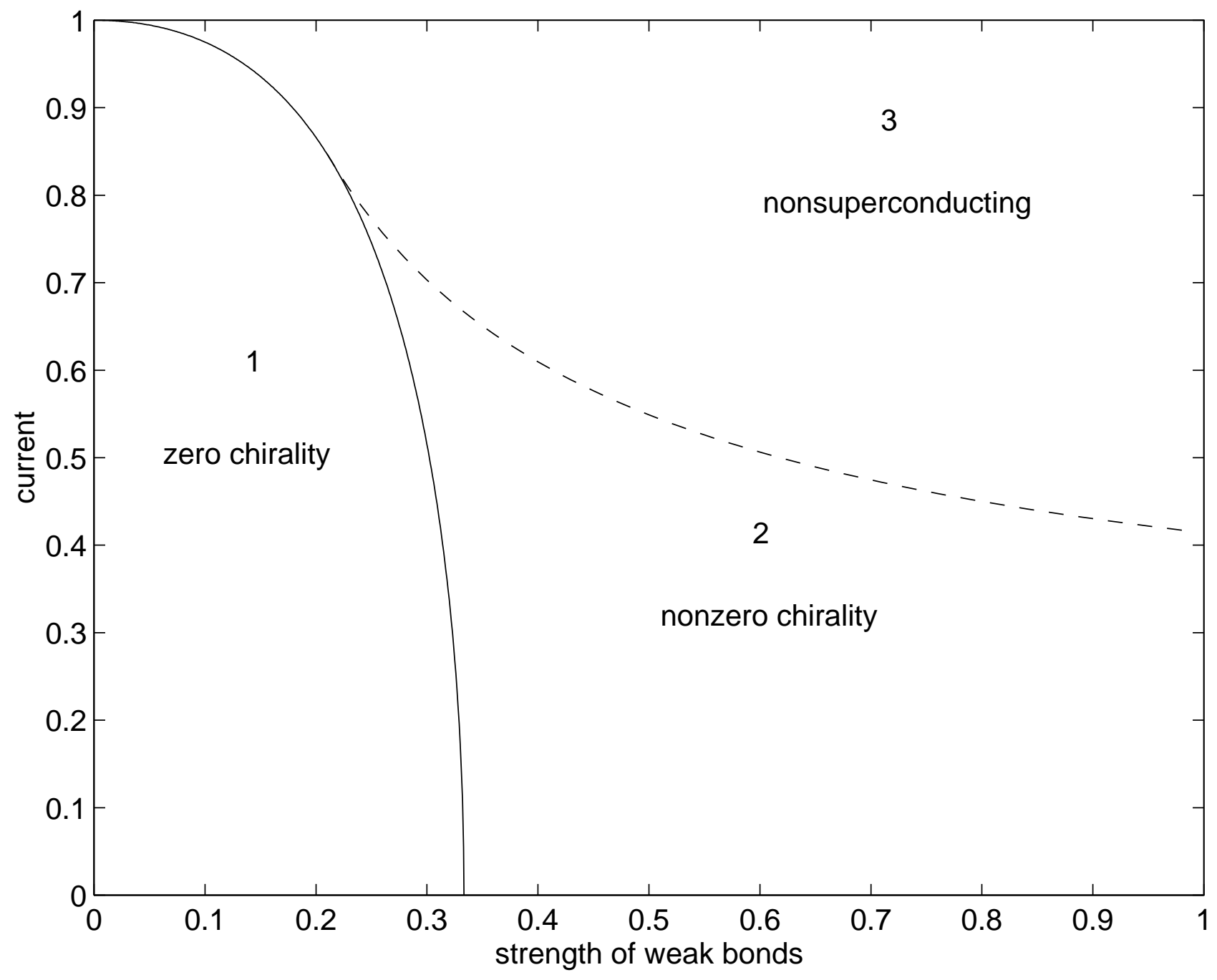




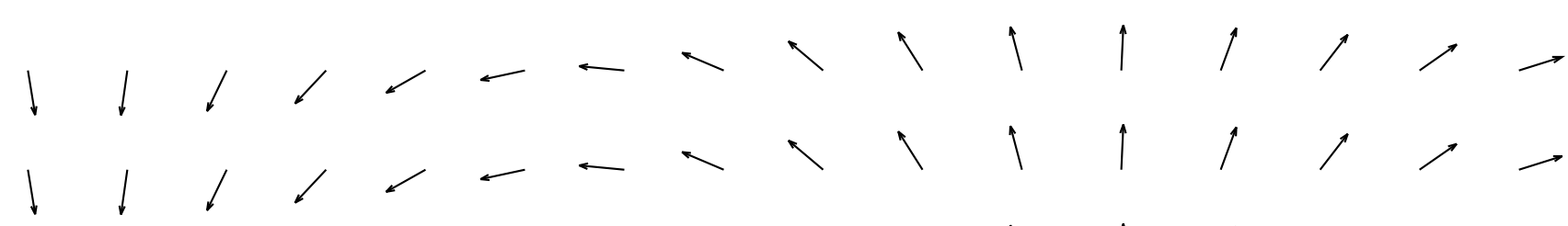

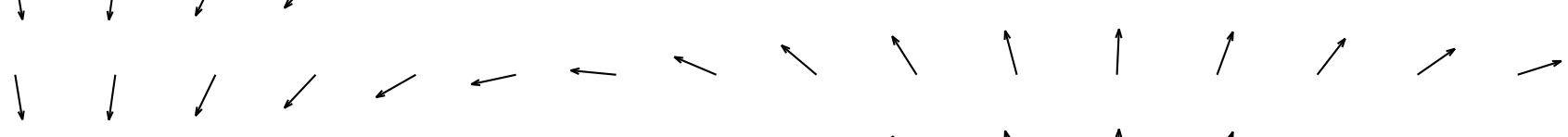

$$
\begin{aligned}
& 11,1, \ldots 1111,1,
\end{aligned}
$$

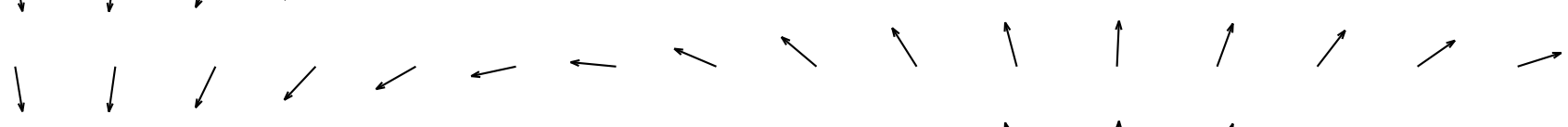

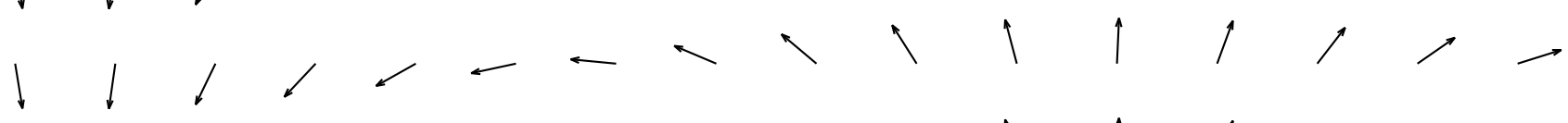

$$
\begin{aligned}
& 11,1, \ldots 1111,1,
\end{aligned}
$$

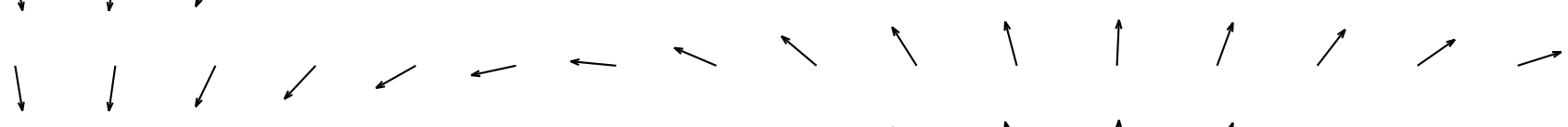

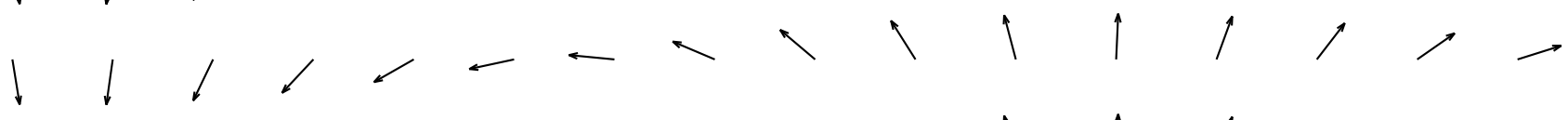

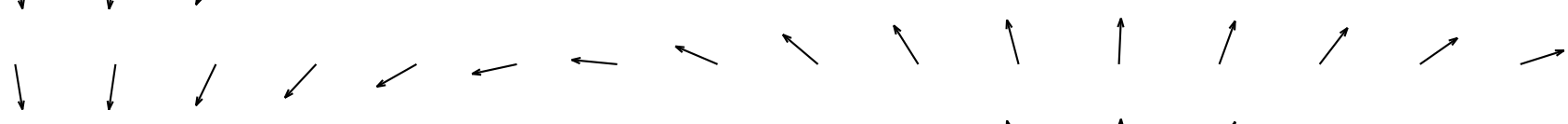

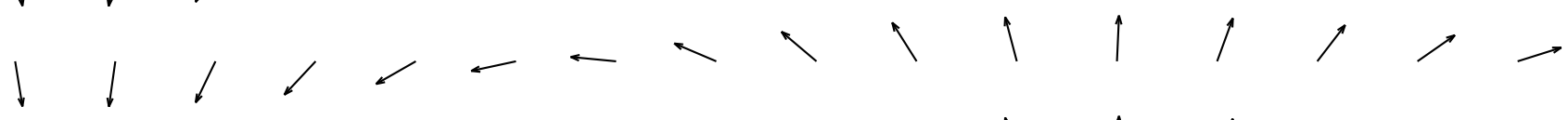

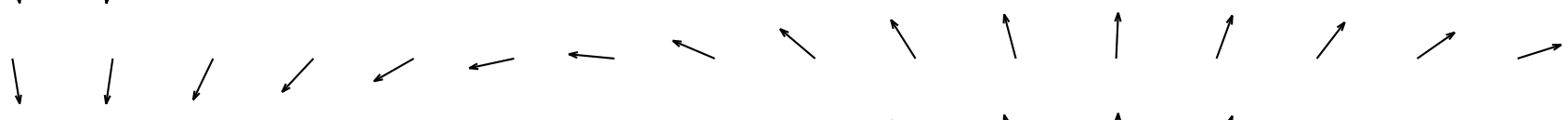

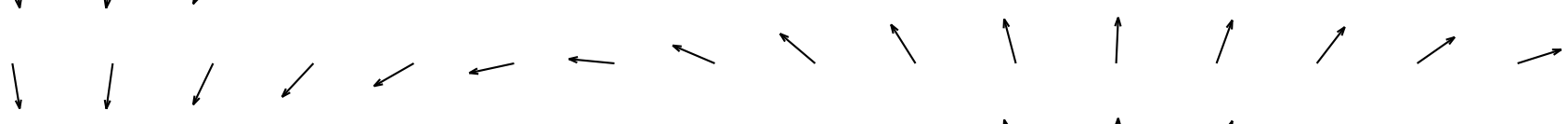

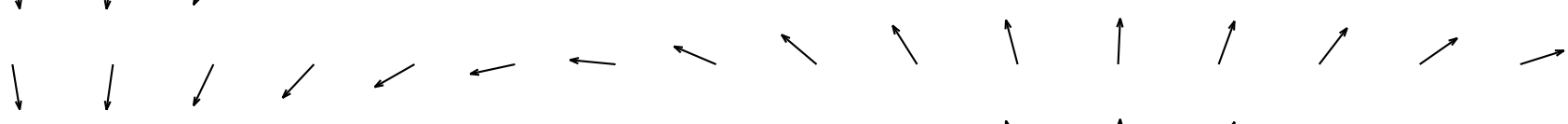

$$
\begin{aligned}
& 11,1,-1,111,1, \\
& 11,1, \ldots 1111,1,
\end{aligned}
$$




$$
\begin{aligned}
& ->11,-111<-\cdots 1 \\
& -1,1,11,1,-1 \\
& \rightarrow-1,1<11<->1 \\
& ->11,+11,-\infty 1 \\
& \rightarrow-1,1<1,->1 \\
& ->11,->11 / 1,-11 \\
& \rightarrow>1,>11<->1 \\
& \rightarrow-11,-111,-11 \\
& \rightarrow>1,->11<1 \\
& \rightarrow-1,1,-111,-11 \\
& \rightarrow>1<1<1<1 \\
& \rightarrow-1,1,1,1,-11 \\
& \rightarrow>1,>11<->1 \\
& \rightarrow-11,+111+-1 \\
& \rightarrow>11,->11<->1 \\
& -1,1,11,-11
\end{aligned}
$$




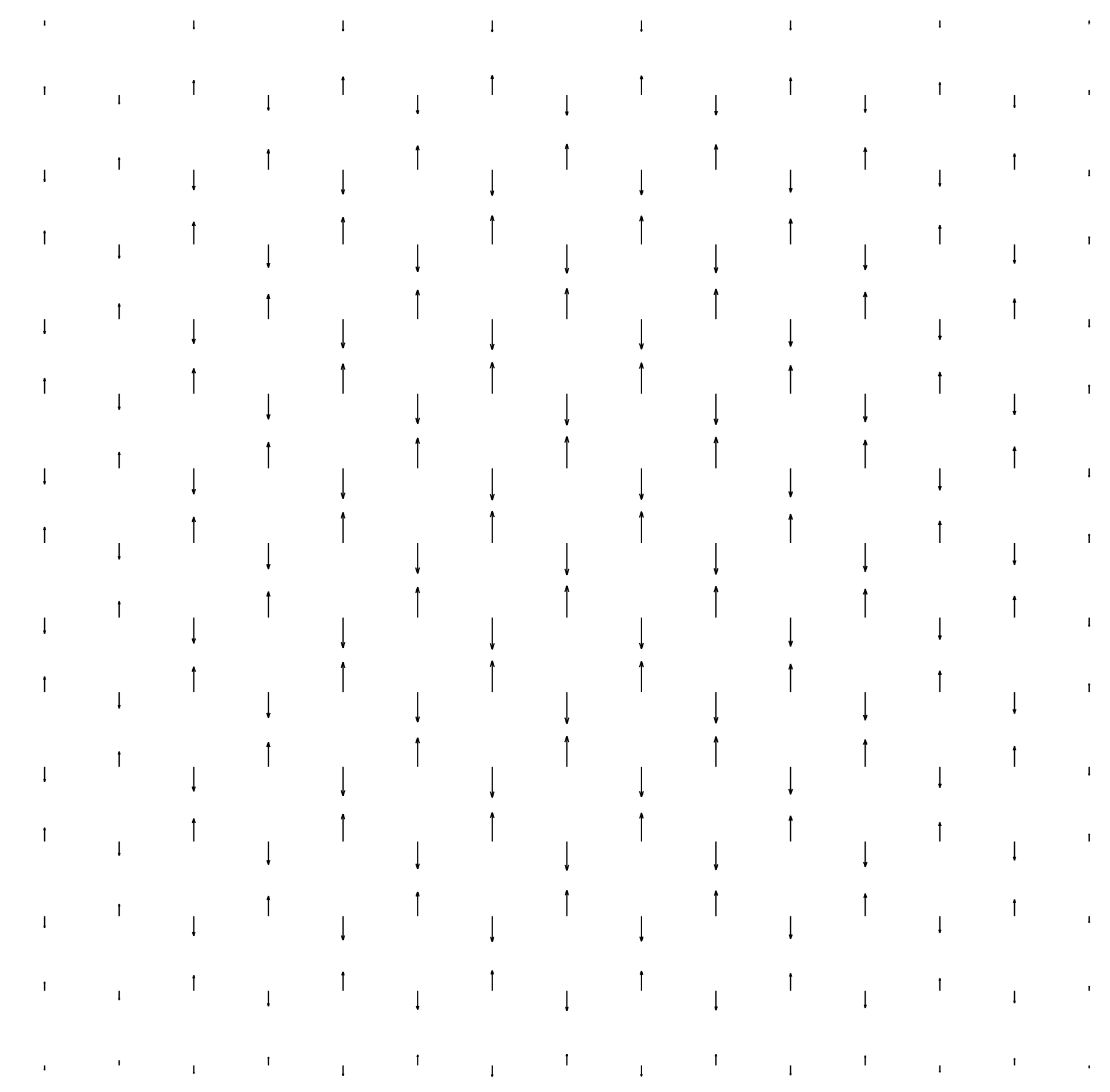

